\title{
Uso tecnopedagógico de dispositivos móviles en la formación de investigadores
}

\author{
Elvia Garduño Teliz
}

\begin{abstract}
Resumen
El objetivo de este trabajo es describir los efectos que tuvo el uso tecnopedagógico de los dispositivos móviles en la formación de diez jóvenes de Educación Media Superior (EMS) y Educación Superior (ES), en dos programas de verano de investigación de EMS y Es, realizados de junio a agosto de 2019 en la Escuela Superior Ciencias de la Educación de la Universidad Autónoma de Guerrero. Los dispositivos móviles se usaron tecnopedagógicamente desde una perspectiva teórica ubicua y conectivista, para la selección del tema, la identificación del objeto de estudio, el planteamiento del problema, la aplicación de la metodología en la búsqueda y recolección de la información, y la presentación de sus avances. El enfoque del trabajo es cualitativo a través de un estudio de caso. A la pregunta ¿cuáles son los efectos del uso tecnopedagógico de los dispositivos móviles en la formación de investigadores?, se responde con resultados sobre el reconocimiento metacognitivo personal a través de una identidad digital, la creación de conexiones, metáforas y relaciones con el proceso de investigación, así como la generación de una comunidad de aprendizaje virtual y ubicua que aplica los principios del conectivismo en la formación de investigadores.
\end{abstract}

Palabras clave: dispositivos móviles, formación, investigación, tecnopedagógico, ubicuidad, personalización, conectivismo.

\section{TECHNO-PEDAGOGICAL USE OF MOBILE DEVICES IN THE TRAINING OF RESEARCHERS}

\begin{abstract}
The aim of this work is to describe the effects that the techno-pedagogical use of mobile devices had on the training of ten young researchers, from Higher Secondary Education (EMS) and Higher Education (ES), in three summer research programs of EMs and ES, carried out from June to August of 2019 at the Higher School of Education Sciences of the Autonomous University of Guerrero.

The mobile devices were used techno-pedagogically from a ubiquitous and connectivist theoretical perspective for topic selection, object of study identification, methodology application in the search and collection of information, and the presentation of the information produced. The approach is qualitative, through a case study. To the question what are the effects of the techno-pedagogical use of mobile devices in the training of researchers?, we respond with results on personal metacognitive recognition through a digital identity, the creation of connections, metaphors and relationships in the research process, as well as the generation of a virtual and ubiquitous learning community, that applies the principles of connectivism in the training of researchers.
\end{abstract}

Keywords: mobile devices, training, research, techno-pedagogical, ubiquity, personalization, connectivism.

Recepción: 06/01/2017. Aprobación: 01/09/2020.

Dol: http://doi.org/10.22201/cuaieed.16076079e.2020.21.6.8 


\section{Elvia Garduño Teliz}

elvia_garduno_teliz@hotmail.com orcid.org/0000-0002-5971-4003

http://tecnologiaseducativasuagro.blogspot.mx

Doctora en Pedagogía por la Universidad Nacional Autónoma de México (UNAM), adscrita a la Universidad Autónoma de Guerrero, perfil PRODEP, candidata al Sistema Nacional de Investigadores. Sus líneas de investigación son tecnopedagogía, personalización del aprendizaje, aprendizaje móvil e inclusión. Gestora tecnopedagógica de plataformas educativas virtuales institucionales, Cursos Masivos Abiertos en Línea y blogs. Integrante del equipo de investigación del proyecto CONACYT-IBERO "La educación durante el covid-19: un análisis desde lo pedagógico, psicológico y tecnológico".

\section{Introducción}

Los dispositivos móviles se han convertido en parte fundamental de nuestra vida diaria. La encuesta nacional de consumo de contenidos audiovisuales 2018 (INEGI, 2018) reflejó entre sus datos que el teléfono celular es el dispositivo que más usan los mexicanos para consumir contenidos por internet, con81\%; la computadora o laptop representa $20 \%$ y las tabletas alcanzan 10\%. También se encontró que del total de personas que utilizan internet en el país, 8 de cada 10 usan redes sociales y mensajería instantánea, encabezadas por las aplicaciones de WhatsApp (86\%) y Facebook (76\%).

Además, la unEsco considera una línea de trabajo e investigación de carácter tecnopedagógico a través de los dispositivos moviles, que se concreta al aplicar ecosistemas de aprendizaje móvil, que integran aplicaciones (apps), contenido y rutas de aprendizaje móvil (Miao, West, So, y Toh, 2017). Por esta razón, es importante considerar el potencial formativo de los dispositivos móviles, no sólo para el desarrollo de habilidades digitales, sino también para la generación de nuevos entornos de conocimiento, mediante la adecuación de contenidos (Cantillo Valero, Roura Redondo y Sánchez Palacín, 2012); la potenciación de las interacciones; la estimulación de la exploración; la comunicación; y el pensamiento crítico y reflexivo (Basantes, Naranjo, Gallegos, y Benítez, 2017); así como el desarrollo de las habilidades cognitivas como solución de problemas, toma de decisiones y el pensamiento creativo (Ramos-Elizondo, Herrera-Bernal y Ramírez-Montoya, 2010). 
"Uso tecnopedagógico de dispositivos móviles en la formación de investigadores"

Elvia Garduño Teliz

Vol. 21, Núm. 6, noviembre-diciembre 2020

Revista Digital Universitaria

En la formación de jóvenes en la investigación, el uso del teléfono celular puede aplicarse en la comprensión de un proyecto de investigación de una manera creativa y reflexiva. Se plantea que a través del uso del celular los estudiantes conecten "Ios nodos o fuentes de información [...] para desaprender, reaprender y aprender el conocimiento como resultado de explorar las ideas del otro en la red de aprendizaje" (Belle, 2019, p. 41). Una característica de los dispositivos móviles es la ubicuidad, es decir, la "disponibilidad de información a cualquier hora y desde cualquier parte [...] lo cual ha de modificar la manera en que se experimenta el mundo, y cómo se enseña" (Vázquez-Cano, Sevillano García, 2015, p.21).

La formación de investigadores es imprescindible para el país y a la vez desafiante, pues involucra algo más que aprender estructuras y conceptos, ya que quien aprende investigación debe hacerlo investigando; por ejemplo al "enseñar a plantear el problema de investigación si se problematiza con quien se inicia en el quehacer científico da mejores resultados que si se da la definición del problema de investigación" (Sánchez, 2014, p.37). Las universidades han recurrido a la generación de programas de verano en el que los participantes tanto de ems como es conviven y aprenden al lado de un investigador reconocido para apoyarlo en uno de sus proyectos y, en el mejor de los casos, realizan desde sus inicios un trabajo propio de investigación. En este último caso, se desarrolló el presente trabajo para dar respuesta a la pregunta de investigación: ¿cuáles son los efectos del uso tecnopedagógico de los dispositivos móviles en la formación de investigadores?

El uso de dispositivos móviles tiene un potencial para el aprendizaje, por lo que el hecho de que éstos sean prohibidos o ignorados en sistemas formales de educación constituye una pérdida de oportunidad en la formación (West y Vosloo, 2013). El aprendizaje móvil va más allá de aplicar los dispositivos (teléfonos celulares, tabletas, iPads, entre otros) en los procesos formativos, pues también se define como "la habilidad de los aprendientes para acceder a la información independientemente del tiempo y del espacio a través de estos dispositivos, así como la administración de su propio proceso de aprendizaje con base en sus diferencias y necesidades individuales" (Talan, 2020, p.80).

En una realidad en la que los teléfonos celulares van adquiriendo cada vez más protagonismo para situaciones cotidianas, es menester aprovechar su conectividad y ubicuidad para empoderar a los usuarios hacia el desarrollo de habilidades relacionadas con la gestión de información, y el pensamiento investigativo y creativo (Rincón Trujillo, Malagón Ruíz, Vasco Gutiérrez y Bedoya Yustres, 2017). En este artículo se propone un uso tecnopedagógico de los teléfonos celulares en la formación de investigadores, al integrar la tecnología, a través de las aplicaciones móviles (apps); la didáctica, al estructurar actividades orientadas a la construcción reflexiva y metafórica de los elementos de la investigación; y la pedagogía, al promover actividades conectivistas y ubicuas para la formación. En este sentido, la labor del investigador-asesor fue la de 
facilitar diversos entornos para promover interacciones, tanto individuales como colaborativas, en la construcción de un proyecto de investigación.

Desde la perspectiva conectivista, las "interacciones sociales están mediadas cada vez más por la tecnología" (Vadillo, 2011, p. 43), por lo que a la par de las habilidades investigativas relativas a procesos de problematización, teorización y comprobación (Rivera y Mondéjar, 2014; Herrera, Fernández y Horta, 2012), también se desarrollaron habilidades digitales "para colaborar en este tipo de redes [...] lo que quizá represente la mayor barrera" (Vadillo, 2011, p. 43).

Desde el aprendizaje ubicuo, se generó un ecosistema para el aprendizaje permanente a través del teléfono celular, el cual favoreció la formación, la relación y la interacción (Quicios García, Ortega Sánchez y Trillo Miravalles, 2015), de tal manera que se facilitaron las integraciones entre los entornos presenciales y los virtuales.

Los entornos de formación presenciales fueron los espacios formales, tales como la sala de videoconferencia, los cubículos de asesoría, el centro de cómputo, la cafetería, la biblioteca y los espacios recreativos. Los espacios informales fueron, por ejemplo, los museos de arte, historia y ciencia, las visitas al centro histórico y al zoológico de la ciudad de Chilpancingo, Guerrero, en México. Los entornos de formación virtuales fueron las aplicaciones de WhatsApp, Bitmoji, Picktochart, Canva, además de las de audio, cámara y video.

\section{Metodología y análisis cualitativo}

Para dar respuesta a la pregunta y lograr el objetivo de la investigación, se empleó el estudio de caso de los diez jóvenes de EMS y ES que realizaron por primera vez una estancia de verano de investigación de junio a agosto de 2019. En EMs fueron cinco participantes de entre 15 y 16 años, tres mujeres y dos hombres provenientes de la Costa Grande del estado de Guerrero; ninguno de ellos había cursado la asignatura de investigación en su bachillerato. En Es fueron cinco participantes de entre 22 y 30 años, tres mujeres y dos hombres provenientes de la ciudad de Chilpancingo, Guerrero, de la Licenciatura Ciencias de la Educación. Todos ellos habían cursado asignaturas relativas a la investigación y tuvieron que elaborar un protocolo para ser aceptados en el programa de verano. A continuación se describen las acciones tecnopedagógicas que realizaron los jóvenes en formación.

De manera previa, los participantes realizaron con sus celulares una lección digital síncrona, que contenía, entre otros aspectos, un diagnóstico sobre los usos y el manejo que hacían de sus aplicaciones en dispositivos móviles, posibles usos del celular para el aprendizaje y algunas ideas para conectar aspectos personales con la investigación. La intención formativa de esta actividad fue comprender el planteamiento conectivista y ubicuo del aprendizaje móvil, para la personalización de sus procesos de investigación. Para ello usaron la aplicación Nearpod. 
"Uso tecnopedagógico de dispositivos móviles en la formación de investigadores"

Elvia Garduño Teliz

Vol. 21, Núm. 6, noviembre-diciembre 2020

Revista Digital Universitaria

En la selección del tema, los jóvenes realizaron en su celular una ruta digital de investigación a través de una infografía. Dicha ruta fue diferenciada para ems y ES, (ver figuras 1 y 2). La intención formativa fue conectar la identidad y aspectos personales (intereses, necesidades, expectativas y pasiones) con el proceso de su investigación. Para ello, usaron las aplicaciones de Bitmoji, Picktochart y Canva.

Para la identificación del objeto de estudio y el planteamiento del problema, los jóvenes buscaron información en las tesis almacenadas en la biblioteca escolar, para identificar dichos elementos y tratar de diferenciarlos. Además, recorrieron entornos presenciales donde fotografiaron, grabaron, ubicaron o postearon información relativa a la investigación. La intención formativa fue generar contenidos digitales ubicuos, para representar el objeto de estudio y el planteamiento del problema desde la metaforización. En estas actividades, se usaron aplicaciones para fotografía, captura de pantalla, edición de imágenes y video, ubicación, fecha y hora, notas de voz y de texto.

En la metodología para la búsqueda y recogida de información, los jóvenes contaron con materiales digitales como videos, sitios web, una webquest, artículos, blogs, cuadros comparativos y ejemplos sobre los paradigmas, enfoques y tipos de investigación, a partir de los cuáles se hicieron ejercicios relativos a identificar qué y cómo se haría para atender la metodología elegida. Se trabajó desde la ubicuidad en la recogida de información para documentar entrevistas, observaciones, videos, encuestas a través de formularios, además de la búsqueda avanzada en la web. Adicionalmente, se compartieron y realimentaron los avances obtenidos de manera presencial y móvil. La intención formativa fue aplicar el dispositivo móvil en la gestión de información (búsqueda, selección, citación y referenciación, publicación, y difusión) del proceso de su investigación. Las aplicaciones utilizadas fueron WhatsApp, Google Scholar, los navegadores y las aplicaciones para fotografía, captura de pantalla, edición de imágenes y video, ubicación, notas de voz y de texto.

Para la presentación de los avances se utilizaron páginas para diseñar presentaciones electrónicas, videos relativos a cómo hablar y controlar los nervios en público, la forma de presentar los resultados de investigación, y recomendaciones para exponer. Además, se aplicaron técnicas de mindfulness para la atención plena, las cuales también se compartieron en audios. Tanto en la construcción de la presentación como en los ensayos para la oralidad se usaron los dispositivos móviles al grabar, temporalizar y realimentar los desempeños. La intención formativa fue desarrollar habilidades para presentar los avances de la investigación realizada con el apoyo de estos dispositivos. Las aplicaciones utilizadas fueron WhatsApp, YouTube, los navegadores, cámara fotográfica, grabación de video y cronómetro.

De todas las acciones anteriores, se evidenciaron interacciones, infografías y videos, que se analizaron para "proporcionar una descripción detallada del caso, un análisis de los temas o asuntos y las interpretaciones o afirmaciones del 
investigador, comentarios auténticos y representativos de los participantes [...] para ilustrar su complejidad" (McMillan y Schumacher, 2005, p.45). Para describir el comportamiento del caso "con sus peculiaridades y circunstancias" (Martínez, 2007), se utilizó el software cualitativo Atlas.ti en su versión 8.4. Se analizaron 30 documentos entre los que se encuentran videos, imágenes, e interacciones en el grupo de chat de WhatsApp. Los códigos obtenidos se agruparon en cuatro categorías, relacionadas con las buenas prácticas en el uso de los dispositivos móviles tomados y adaptados del Plan Ceibal (Rivera y Cobo, 2018), a saber:

Ubicuidad. Se refiere a la inmersión, por lo que es penetrante, omnipresente para aprender en cualquier lugar y en cualquier momento con la ayuda del dispositivo móvil (Jones y Jo, 2004, párrs. 2-5). Se registraron ocho códigos: ambientes de aprendizaje, aplicaciones, arte, contexto, dificultades, dispositivos móviles, fuentes de información e historia.

Conectivismo. Se refiere al enfoque pedagógico que conecta personas, nodos, fuentes de información para abrir "espacios para el diálogo e intercambio social entre los individuos" (Cortez, 2019, párr. 7). Así, el aprendizaje se da a través del uso de aplicaciones móviles. Se registraron 12 códigos: aplicaciones, colaboración, conectividad, fuentes de información, gestión de información, recursos, sujetos, supuestos de investigación y tıc.

Personalización. Se ubican las decisiones de los usuarios respecto a su propio aprendizaje, en tiempo real y con apoyo de los dispositivos móviles. Algunos autores como Pen, Sanshan y Spector (2019) lo consideran ligado al aprendizaje adaptativo, pues generara actividades flexibles que motivarán al compromiso y a su vez serán realimentadas en el proceso. El aprendizaje personalizado presta especial atención a los conocimientos previos, las necesidades, las capacidades y las percepciones de los estudiantes durante los procesos de enseñanza y aprendizaje. Se trata, por lo tanto, de una formación centrada en el alumno (oIE-UNESCO, 2017, p.5). Se registraron 21 códigos: apoyo entre sí, aprendizajes, asignaturas preferidas, carrera profesional a elegir (en el caso de Ems), conocimientos, efectos, emociones, expectativas, fortalezas, ideas, identidad, intereses, logros y deseos, metáforas, motivación, pasiones, perspectivas, recursos, relaciones, resolución de dudas y valoraciones.

Investigación tecnopedagógica. Se refiere al uso pedagógico de las tecnologías móviles para el desarrollo de los procesos y habilidades de investigación, pues integra estrategias, ambientes virtuales y competencias investigativas (Landazábal, Páez y Pineda, 2013). Se registraron 15 códigos: actividades tecnopedagógicas, apoyo entre ellos, autoría, gestión de información, institución universitaria, logística, materiales, objeto de estudio, problema, proceso de la investigación, resultados, revisiones, tema, valoración y variables.

Como resultado del análisis e interpretación cualitativa de las categorías anteriores, se encontraron diversos efectos del uso tecnopedagógico de los 
dispositivos móviles en la formación de investigadores, los cuales se presentan y diferencían de conformidad con el nivel educativo de los participantes.

El efecto personalización. En Ems se encontraron altas expectativas sobre la investigación, relativas a mejorar los trabajos, aprender algo nuevo, y clarificar la elección de su carrera profesional. Este primer problema cognitivo fue abordado en la construcción de su ruta de investigación (ver figura 1), al definir su identidad - "soy buena aconsejando y enfocarme en todo" (E1), "soy honesta, a veces responsable, soy muy sentimental..." (E2) - , sus pasiones, gustos, habilidades e intereses vocacionales. En estas rutas, se observaron rasgos metafóricos que denotan sentimientos positivos asociados a la experiencia de aprender a investigar, errores de ortografía, y una incipiente experiencia en el uso de TIc.

Figura 1. Ejemplo de ruta de investigación de Ems.

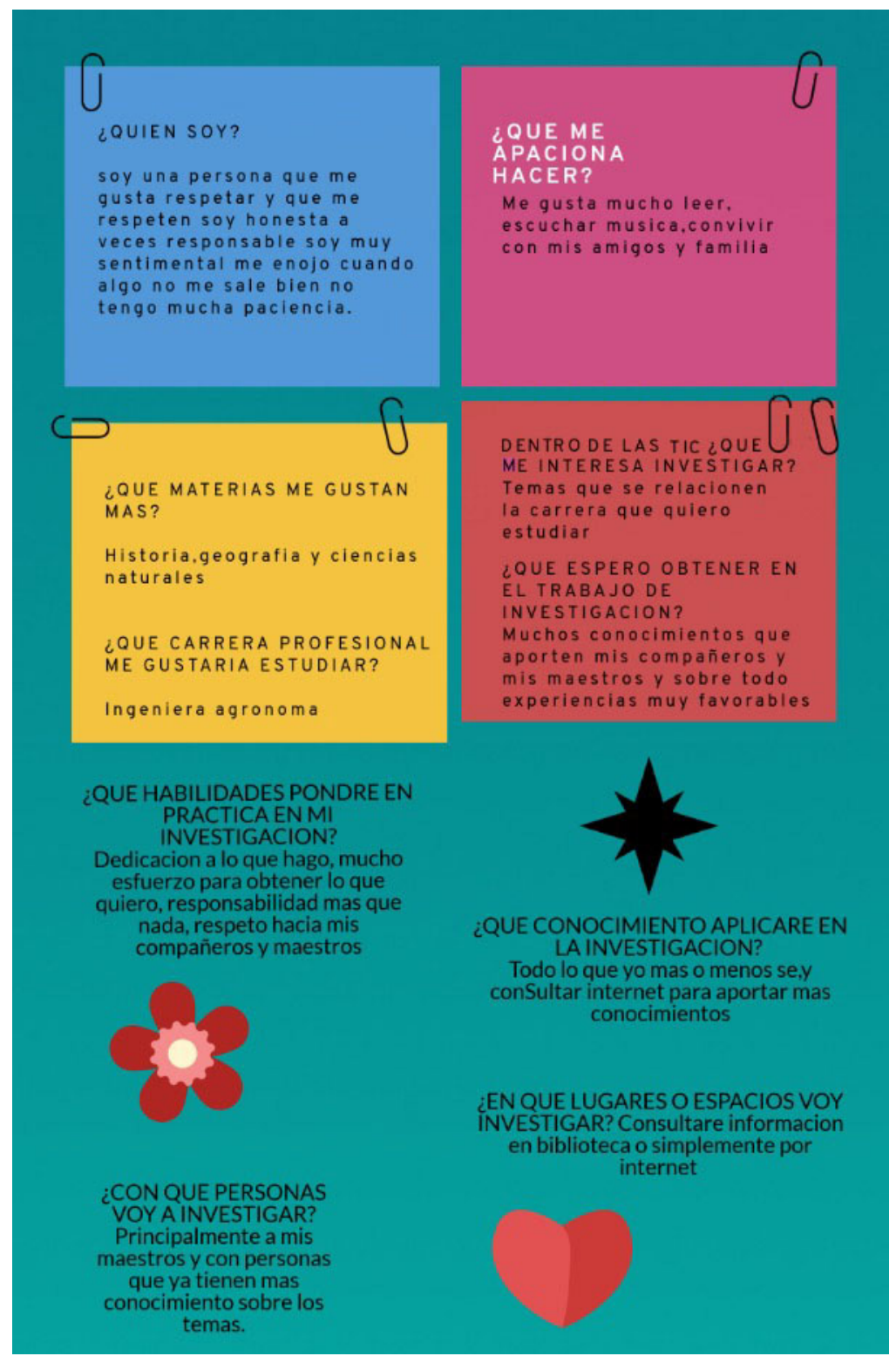


En el caso de Es, los intereses y las expectativas de los estudiantes están claramente identificadas dentro del ámbito de las Tıc (ver figura 2), al mencionar, por ejemplo, "la tecnología móvil, la mejora en el desempeño de la investigación" (E3), "conocer y aplicar las tic" (E4), "buscar dar una respuesta satisfactoria a la investigación" (E3), "aprender a investigar" (E5), "mejorar mi habilidad para buscar información [...] comunicar ideas a personas de diferente edad" (E6). Lo que se desea lograr con el trabajo trasciende los intereses personales y académicos al "generar un posible cambio [...] creer como estudiante" (E3), o "lograr la concientización [...] y disminuir el analfabetismo digital" (E4). El verbalizar estas ideas constituye un punto de partida para visualizar el planteamiento del problema, pues es menester del investigador "convertir problemas cotidianos en problemas investigativos y llegar a conclusiones válidas tomadas de la reflexión sobre su propia experiencia" (Quintero, Ancízar y Múnevar, 2008, p.36).

Tanto en metodología tecnopedagógica de este trabajo como en los resultados del análisis, se muestra la aplicación de un enfoque centrado en el aprendiente -en este caso los investigadores en formación-, por lo que al construir su ruta de investigación "tienen la voz y eligen el cómo aprenden mejor y cómo demostrar lo que ya saben, así como la forma adecuada de adquirir conocimiento de acuerdo a sus necesidades e intereses" (Friend, Patrick, Schneider, y Vander 2017, p.5).

Todos los participantes tuvieron dificultades para relacionar sus pasiones con los temas u objetos de estudio de la investigación. Lo cual posiblemente sea el resultado de procesos de formación estandarizados y perceptivos. "Las pasiones son las rutas y filtros para desarrollar un aprendizaje que permanecerá en las mentes de los alumnos, será valioso en sus vidas y les hará querer aprender más" (Prensky, 2013, p.75), de ahí su importancia en este trabajo. Los participantes visualizan un arquetipo del investigador como un indagador o detective, más que como un científico. Por ende, se relacionan los artefactos como la lupa, el foco, el lápiz, la computadora, el dispositivo móvil, el libro, como fuentes inspiradoras de ideas y de gestión de la información. Se denotan emociones positivas ligadas a la colaboración y al trabajo personal y con otros, así como compromisos personales al invertir el tiempo, paciencia, esfuerzo y la curiosidad.

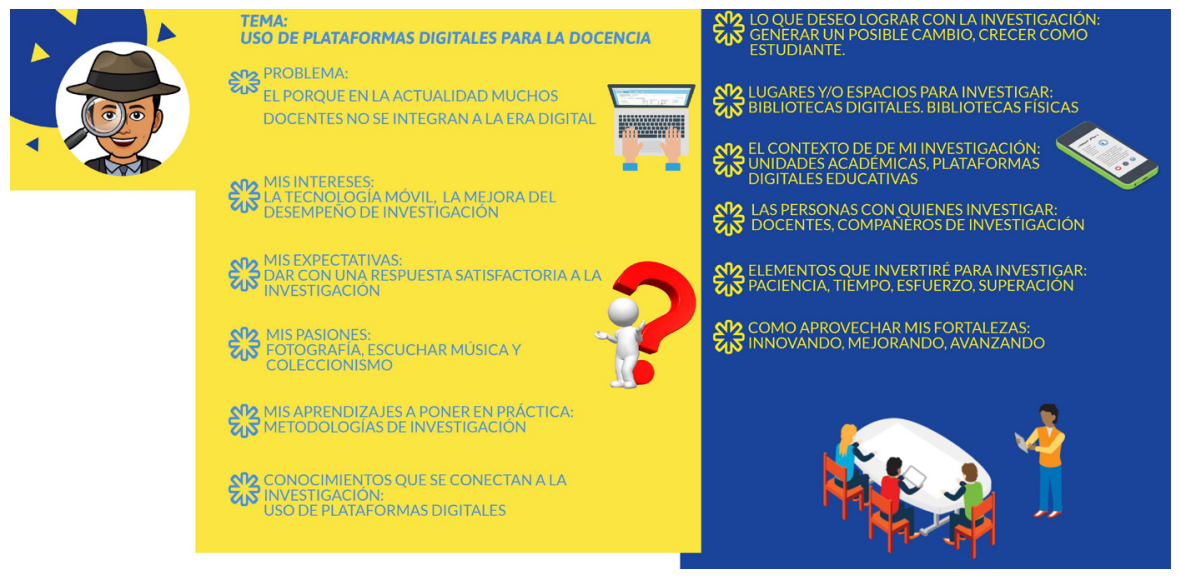


Figura 3. Estas imágenes reflejan distintos procesos de metaforización de objetos de estudio. De izquiera a derecha, la primera imagen es el resultado de una búsqueda en la web, la segunda imagen muestra un collage de fotografías tomadas por la participante en el centro de la Ciudad de Chilpancingo, Guerrero, México. En tanto, las últimas tres imágenes fueron fotografiadas por los participantes en el Museo Universitario José Juárez en la exposición "Sintomatologías" por el maestro Everardo Reyes.
El efecto ubicuidad. Se constató al interrelacionar metafóricamente lo encontrado en los entornos presenciales y virtuales, como fuentes de inspiración ubicua para generar ideas, "A primera vista consideré que no podría encontrar nada que pudiera ayudar a mi tema de investigación, pero conforme se fue avanzando en el recorrido descubrí que se tiene que ir más allá de lo que está a simple vista, lo cual es con lo primero que me encontré" (E8) (ver figura 3).
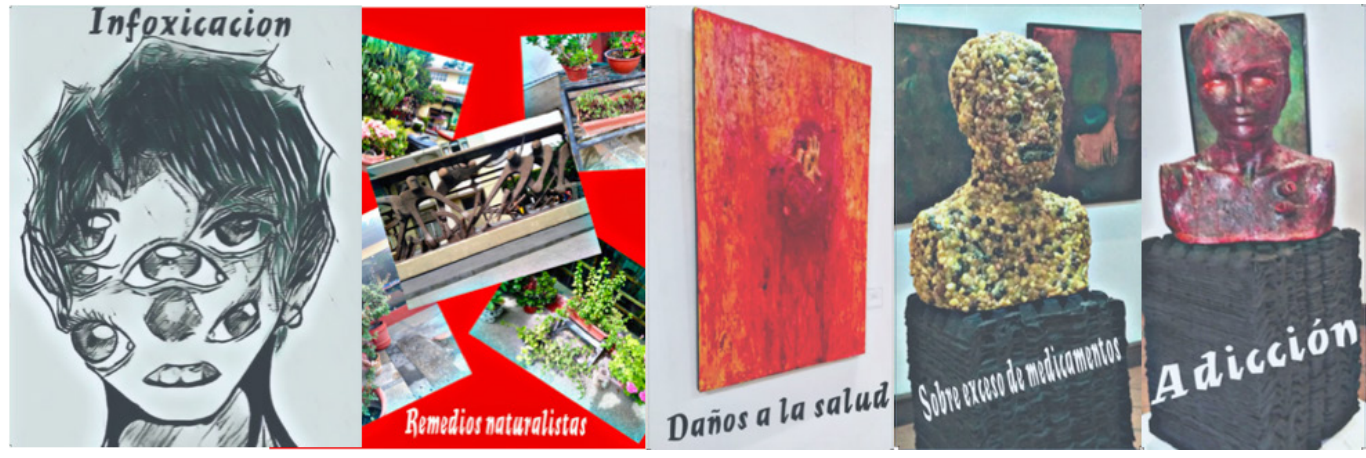

La ubicuidad incidió en la mejor comprensión del tema y del objeto de estudio, así como en el reconocimiento de los espacios informales como fuentes de información. En particular, el arte tuvo una incidencia en el pensamiento creativo de los participantes a través de la metaforización, la relación imaginativa, y la crítica entre lo que se observa, se comprende y se investiga, pues "la creatividad domina un proceso de hacer o producir, la criticidad uno de evaluar y juzgar [...] por lo que el pensamiento profundo requiere de imaginación y de la disciplina intelectual" (Paul y Elder, 2005, p.13). Las fotografías y videos documentaron el reconocimiento de los artefactos socioculturales como elementos relacionados con la investigación, surgidos la espontaneidad de lo ubicuo, y no de la prescripción del profesor que enseña a investigar, lo cual atiende múltiples perspectivas a través de las cuáles un problema de investigación puede ser abordado. Ante estos hallazgos se precisa considerar "que los estudiantes necesitan moverse ubicuamente a partir de objetivos de aprendizaje que los orienten" (Li, Chiu, y Tseng, 2019), por lo que las actividades ubicuas tuvieron una intencionalidad formativa.

El efecto colaboración. Se encontró un creciente apoyo entre colegas y con otros investigadores a través de la web, emociones positivas relacionadas con la confianza y el manejo de la incertidumbre, una mejor comunicación e interacción en la logística del trabajo al compartir materiales, evidencias, contactos, software, horarios, asesorías individuales y grupales, así como las emociones previas a la presentación de sus avances de investigación en los coloquios de investigación. Por lo tanto, se infiere que se generó una comunidad de aprendizaje virtual y ubicua, que incidió en el sentido de solidaridad, empatía e intercambios para sus proyectos de investigación:

30/7/19 7:43 p. m. - Ruth Ss: Buenas tardes compañeros, todavía falta que me envien su actividad conforme a la visita del museo de la avispa. Por favor les pido su apoyo para que pueda seguir trabajando en mi investigación

30/7/19 7:52 p. m. - Miriam: En un rato te las envío "n 
"Uso tecnopedagógico de dispositivos móviles en la formación de investigadores"

Elvia Garduño Teliz

Vol. 21, Núm. 6, noviembre-diciembre 2020

Revista Digital Universitaria

El efecto conectivista. En todo el proceso, se evidenció la aplicación de los principios conectivistas establecidos por Siemens (2004), que se precisan a continuación.

a. El aprendizaje y el conocimiento dependen de la diversidad de opiniones. A través de las rutas se personalizó el proceso de la investigación; las aplicaciones móviles permitieron generar interacciones virtuales, que enriquecieron las presenciales al compartir imágenes, dudas, ubicaciones, acciones, evidencias de avances y realimentaciones.

b. El aprendizaje es un proceso de conectar nodos o fuentes de información especializados. Al realizar procesos de gestión de información de manera ubicua, los jóvenes hallaron otros lugares para aprender a investigar. Además de la web y la escuela, el grupo de WhatsApp se convirtió en un repositorio de materiales, evidencias, imágenes, notas y recomendaciones de fuentes de información.

c. El aprendizaje puede residir en dispositivos no humanos. Los teléfonos celulares y las apps se constituyeron como espacios y oportunidades de aprendizaje mediante un uso intencionado, durante el proceso de investigación, a través de las actividades tecnopedagógicas.

d. La capacidad de saber más es más crítica que aquello que se sabe en un momento dado. Al desarrollar la gestión de información en diferentes fuentes y al concretarla en la estructura y presentación de su investigación, los estudiantes centraron sus habilidades en la búsqueda, selección y publicación de la información como parte central del proceso de la investigación. No obstante, hubo debilidades en la búsqueda avanzada en la web, en el uso del formato APA y en la comprensión significativa de los aspectos metodológicos de la investigación. A pesar de ello, a través de los dispositivos móviles se motivó la construcción de relaciones significativas inherentes a la capacidad de saber investigar.

e. La alimentación y mantenimiento de las conexiones es necesaria para facilitar el aprendizaje continuo. Aunque estas actividades tecnopedagógicas fueron significativas para los estudiantes, no se tiene certeza si les darán continuidad. No obstante, se han sentado las bases para nuevas experiencias de aprendizaje.

f. La habilidad de ver conexiones entre áreas, ideas y conceptos es una habilidad clave. La metaforización de las actividades ubicuas para identificar relaciones con los procesos de la investigación presentó cierta dificultad, pues los jóvenes están habituados a recibir conceptos y pasos a seguir en sus procesos formativos.

g. La actualización del conocimiento es la intención de todas las actividades conectivistas de aprendizaje. La posible continuidad del proceso de investigación o la transferencia de estas habilidades a otros contextos de aprendizaje por los jóvenes es una oportunidad para seguir este principio. 
"Uso tecnopedagógico de dispositivos móviles en la formación de investigadores"

Elvia Garduño Teliz

Vol. 21, Núm. 6, noviembre-diciembre 2020

Revista Digital Universitaria

h. La toma de decisiones es, en sí misma, un proceso de aprendizaje. El acto de decidir desde el principio hasta el final de este proceso fue el mayor reto y éxito de las actividades, dado que los estudiantes decidieron el qué y cómo investigar, y generaron conexiones sobre estas decisiones.

Finalmente, estas experiencias incidieron en las posibilidades de dar continuidad al proceso de la investigación para futuros trabajos de tesis, en el caso de los jóvenes de educación superior.

\section{Conclusiones}

El uso de los teléfonos celulares en la formación de investigadores obliga a replantear la forma en la que se enseña a investigar. En este trabajo, se llevaron a cabo actividades tecnopedagógicas, en las que el uso de aplicaciones móviles conectó ideas, intereses, necesidades, expectativas y pasiones de los estudiantes con el proceso de investigación. Entre estas actividades se encuentran la generación de su identidad como investigadores, creación de su ruta de investigación, identificación del tema, construcción de su objeto de estudio, planteamiento del problema y la metodología, y la presentación de avances preliminares de la investigación.

A través del análisis cualitativo se logró el objetivo de trabajo y se respondió la pregunta de investigación, pues se describieron los efectos que tuvo el uso tecnopedagógico de los dispositivos móviles en la formación de investigadores, los cuáles fueron:

El efecto personalización. Al establecer una identidad como investigadores, a través del reconocimiento metacognitivo personal y la creación de conexiones con el tema y el objeto de estudio.

El efecto ubicuidad. Con la construcción de relaciones y metáforas para la problematización y la redacción de preguntas de investigación.

El efecto colaboración. Al generar una una comunidad de aprendizaje virtual y ubicua, evidenciada a través del grupo de WhatsApp, lo que favoreció a la integración, participación y realimentación del proceso de la investigación para la aplicación de la metodología en el trabajo de campo y la presentación de avances.

El efecto conectivista. A partir de la aplicación práctica de los principios conectivistas establecidos por Siemens (2004), en los que los jóvenes desarrollaron habilidades relativas a conectar nodos o fuentes de información, reconocer el potencial de los dispositivos móviles para su formación en la investigación, visualizar conexiones y la toma de decisiones. Las habilidades relativas a la actualización del conocimiento y el mantenimiento de las conexiones quedaron abiertas ante la posibilidad de los estudiantes de dar continuidad al trabajo de investigación. 
Pese a las limitaciones en tiempo y en seguimiento, el presente estudio de caso aporta una metodología tecnopedagógica para la aplicación de los teléfonos celulares en la formación de investigadores, a través del aprendizaje móvil. También contribuye a ampliar las experiencias ubicuas y personalizadas en estos procesos, de tal manera que se puedan replicar en otros contextos formativos. Los usos tecnopedagógicos de los dispositivos móviles y sus efectos en los estudiantes presentan oportunidades para cambiar las formas de enseñanza y aprendizaje de las habilidades investigativas, e incidir en generar interés y gusto por la investigación. En estos contextos de pandemia y de nueva normalidad, se ha demostrado la necesidad de formar en los usos educativos de dispositivos móviles, por lo que toda experiencia que contibuya a ello puede ser replicada, adaptada y mejorada.

\section{Referencias}

* Acharya, A., y Verstak, A. (2004). Google Scholar [sitio web]. https://scholar.google.com

* Basantes, A. V., Naranjo, M. E., Gallegos., M. C.,y Benítez, N. M. (2017). Los dispositivos móviles en el proceso de aprendizaje de la Facultad de Educación Ciencia y Tecnología de la Universidad Técnica del Norte de Ecuador. Formación Universitaria, 10(2), 79-88. Dol: http://dx.doi.org/10.4067/S0718-50062017000200009.

* Jinot, B. L. (2019). An Evaluation of a Key Innovation: Mobile Learning. Academic Journal of Interdisciplinary Studies, 8(2), 39-45. Dol: https://doi.org/10.2478/ajis2019-0014

* Blackstock, J. (2007). Bitmoji [aplicación móvil]. https://play.google.com/store/ apps/details?id=com.bitstrips.imoji

* Cantillo Valero, C., Roura Redondo, M., y Sánchez Palacín, A. (2012, junio). Tendencias actuales en el uso de dispositivos móviles en educación. La educ@ ción Digital Magazine, 147, 1-21. http://www.educoas.org/portal/la_educacion_ digital/147/pdf/ART_UNNED_EN.pdf

* Chin, G., y Zaggi, A. (2012). Picktochart [aplicación web]. https://piktochart.com/

* Cortez, D. (2019, febrero). El uso del M-learning para la enseñanza-aprendizaje en primaria. Revista Atlante: Cuadernos de Educación y Desarrollo. https://www.eumed. net/rev/atlante/2019/02/mlearning-primaria.html

* Facebook. (2009) WhatsApp [aplicación móvil]. https://play.google.com/store/ apps/details?id=com.whatsapp

* Friend, B., Patrick, S., Schneider, C., y Vander, T. (2017). What's Possible with Personalized Learning? An Overview of Personalized Learning for Schools, Families y Communities. International Association for K-12 Online Learning (iNACOL). https://aurora-institute.org/wp-content/uploads/iNACOL_Whats-Possible-withPersonalized-Learning.pdf 
"Uso tecnopedagógico de dispositivos móviles en la formación de investigadores"

Elvia Garduño Teliz

Vol. 21, Núm. 6, noviembre-diciembre 2020

Revista Digital Universitaria

* Herrera Miranda, G. L., Fernández Montequín, Z. de la C. y Horta Muñoz, D. M. (2012). Estrategia para la formación de habilidades investigativas en estudiantes de medicina. Revista de Ciencias Médicas de Pinar del Río, 16(4), 98-112. http://www. revcmpinar.sld.cu/index.php/publicaciones/article/view/960

* INEGI (2018). Encuesta Nacional Sobre Disponibilidad y Uso de Tecnologías de la Información en los Hogares (ENDUITH). https://www.inegi.org.mx/programas/dutih/2018

* Jones, V. y Jo, J. H. (2004). Ubiquitous learning environment: An adaptive teaching system using ubiquitous technology. En R. Atkinson, C. McBeath, D. Jonas-Dwyer y R. Phillips (Eds.), Beyond the comfort zone: Proceedings of the 21st ascilite Conference (pp. 468-474). http://www.ascilite.org.au/conferences/perth04/procs/jones.html

* Karim, J., Chen, S. y Hurley, Chad (2005) YouTube [sitio web]. https://www.youtube.com/

* Landazábal, D., Páez, D. y Pineda, E. (2013, septiembre-diciembre). Diseño de una innovación pedagógica para la formación en investigación apoyada en ambientes digitales. Revista Virtual Universidad Católica del Norte, (40), 4-30. https://www. redalyc.org/articulo.oa?id=1942/194229200002

* Li, W., Chiu, C. y Tseng, J. C. R. (2019, abril). Effects of a Personalized Navigation Support Approach on Students' Context-Aware Ubiquitous Learning Performances. Journal of Educational Technology \& Society, 22(2), 56-70. Dol: https:// www.doi.org/10.2307/26819617

- Martinez, R. (2007). La investigación en la práctica educativa: Guía metodológica de investigación para el diagnóstico y evaluación en los centros docentes. Ministerio de educación y ciencia.

* Nearpod Inc. (2012) Nearpod [aplicación móvil]. https://play.google.com/store/ apps/details?id=com.panareadigital.Nearpod\&hl=es_MX

* McMillan, J. y Schumacher, S. (2005). Investigación educativa una introducción conceptual. Pearson Education.

- Miao, F., West, M., So, H. y Toh, Y. (2017). Supporting teachers with mobile technology. Lessons drawn from unesco projects in Mexico, Nigeria, Pakistan and Senegal. unEsco. http://unesdoc.unesco.org/images/0025/002515/251511e.pdf

* ole-unesco. (2017). Herramientas de formación para el desarrollo curricular: Aprendizaje personalizado. http://www.eduy21.org/Publicaciones/Aprendizaje\%20 PersonalizadoUnesco.pdf

* Paul, R., y Elder, L. (2005). Estándares de competencia para el pensamiento crítico. Una Guía Para los Educadore en los Estándares, Principios, Desempeño, Indicadoresy Resultados Con una Rúbrica Maestra en el Pensamiento Crítico. Fundación para el pensamiento crítico. https://www.criticalthinking.org/resources/PDF/SPComp_Standards.pdf

* Peng, H., Sanshan, M., y Spector, J. (2019). Personalized Adaptive Learning: An Emerging Pedagogical Approach Enabled by a Smart Learning Environment. Proceedings of 2019 International Conference on Smart Learning Environments. En Chang, M., Popescu, E., N.-S. Kinshuk Chen, M. Jemni, R. Huang, J. M. Spector y D. G. Sampson (Eds.) Foundations and Trends in Smart Learning (pp. 171-176). Springer. https://www.springer.com/gp/book/9789811369070 
"Uso tecnopedagógico de dispositivos móviles en la formación de investigadores"

Elvia Garduño Teliz

Vol. 21, Núm. 6, noviembre-diciembre 2020

Revista Digital Universitaria

* Perkins, M. Obrecht, C., y Adams, C. (2012). Canva [aplicación web]. https://play. google.com/store/apps/details?id=com.canva.editor

* Quicios García, M. del P., Ortega Sánchez, I., y Trillo Miravalles, M. P. (2015). Aprendizaje ubicuo de los nuevos aprendices y brecha digital formativa. PíxelBit. Revista de Medios y Educación, (46), 155-166. Dol: https://doi.org/10.12795/ pixelbit.2015.i46.10

* Quintero-Corzo, J., Múnevar-Molina, R. A., y Munévar-Quintero, F.(2008). Semilleros de investigación: una estrategia para la formación de investigadores. Educación y Educadores, 11(1), 31-42. http://www.scielo.org.co/pdf/eded/v11n1/v11n1a03.pdf

* Ramos-Elizondo, A., Herrera-Bernal, J., y Ramírez-Montoya, M. (2010). Developing cognitive skills with mobile learning: A case study [Desarrollo de habilidades cognitivas con aprendizaje móvil: Un estudio de casos]. Comunicar, 34, 201-209. Dol: https://doi.org/10.3916/C34-2010-03-20

* Rincón Trujillo, H. L., Malagón Ruíz, R. Y., Vasco Gutiérrez, D. F., y Bedoya Yustres, S. (2017). Prácticas pedagógicas mediadas por dispositivos móviles: Estrategias para desarrollar habilidades de pensamiento investigativo y creativo. Rutas De formación: Prácticas y Experiencias, 2, 79-83. Dol: https://doi.org/10.24236/24631388.n2.2016.585

* Rivera García, C. G. y Mondéjar Rodríguez, J. (2014, enero-junio). La lectura crítica como una habilidad investigativa en estudiantes universitarios. Revista Amauta, (23), 163-176. http://investigaciones.uniatlantico.edu.co/revistas/index.php/ Amauta/article/download/1057/699/

* Rivera-Vargas, P., y Cobo Romaní, C. Plan Ceibal en Uruguay: una política pública que conecta inclusión e innovación. En P. Rivera-Vargas, J. Muñoz-Saavedra, R. Morales-Olivares y S. Butendieck-Hijerra (Eds.), Políticas Públicas para la Equidad Social (pp. 13-29). Colección Políticas Públicas, Universidad de Santiago de Chile. https://core.ac.uk/download/pdf/189880651.pdf

- Sánchez, R. (2014). Enseñar a investigar: una didáctica nueva de la investigación en ciencias sociales y humanas. México:IIsuE.

* Siemens, G. (2004). Una teoría de aprendizaje para la era digital. Recuperado de: https://www.comenius.cl/recursos/virtual/minsal_V2/Modulo_1/Recursos/ Lectura/conectivismo_Siemens.pdf

* Talan, T. (2020). The Effect of Mobile Learning on Learning Performance: A MetaAnalysis Study. Educational Sciences: Theory and Practice, 20(1), 79-103. Dol: https:// doi.org/10.12738/jestp.2020.1.006

* Vadillo, G. (2011). Entrevista a George Siemens Desarrollador del Conectivismo. Revista Mexicana de Bachillerato a Distancia, 3(6), 41-47. Dol: http:// dx.doi.org/10.22201/cuaed.20074751e.2011.6.65058

* Vázquez-Cano, E., y Sevillano García, M. (2015). Dispositivos digitales móviles en Educación: El aprendizaje ubicuo. Narcea Ediciones.

* West, M., y Vosloo, S. (2013). Directrices de la unesco para las políticas de aprendizaje móvil. UNESCO. http://www.unesco.org/new/fileadmin/MULTIMEDIA/HQ/ED/ICT/ images/114_13_ED_UNESCO_Policy_Guidelines_for_Mobile_Learning_S.pdf 


\section{Cómo CITAR ESTE ARTículo}

* Garduño Teliz, Elvia. (2020, noviembre-diciembre). Uso tecnopedagógico de dispositivos móviles en la formación de investigadores. Revista Digital Universitaria (RDU), 21(6). Dol: http://doi.org/10.22201/cuaieed.16076079e.2020.21.6.8 